\title{
Supplementary Notes for
}

\section{All-electrical measurement of interfacial \\ Dzyaloshinskii-Moriya interaction using collective spin-wave dynamics}

Jong Min Lee ${ }^{l}$, Chaun Jang ${ }^{l}$, Byoung-Chul Min ${ }^{l, *}$, Seo-Won Lee ${ }^{2}$, Kyung-Jin Lee ${ }^{2}$, and Joonyeon Chang ${ }^{l}$

${ }^{1}$ Center for Spintronics, Korea Institute of Science and Technology, Seoul 136-791, Korea

${ }^{2}$ Department of Materials Science and Engineering and KU-KIST Graduate School of Converging Science and Technology, Korea University, Seoul 136-701, Korea

*Correspondence should be addressed to B-C. M. (min@kist.re.kr) 


\section{Spin-wave excitation from meander-line antennae}

For a quasi-monochromatic excitation of spin wave, we employ a coplanar wave guide (CPW) consisting of five-segment meander lines (See Fig. 1b). The frequency response of the spinwave excitation from the meander-line antenna is given by a complete Fourier transform of current density $\left(\widetilde{j}_{\varepsilon}(k)\right)[1]$ :

$$
\frac{\widetilde{j}_{\varepsilon}(k)}{I}=\frac{\widetilde{j}_{\text {pattern }}(k)}{I}\left[1-2 \cos \left(k L_{\text {pattern }}\right)+2 \cos \left(2 k L_{\text {pattern }}\right)\right] \text {, }
$$

where $\widetilde{j}_{\text {pattern }}(k)$ is the Fourier transform of the linear current density of the elementary CPW pattern (ground-signal-ground lines), $I$ is the magnitude of total current, and $L_{\text {pattern }}$ is the spatial period of meander lines. As shown in Figure S1, the signal from the meander-line antenna presents much narrower linewidth and higher intensity than a single CPW antenna.

\section{Measurement of interface anisotropy}

The interface anisotropy of the samples is determined from $M-H$ hysteresis loops measured by a vibrating sample magnetometer (Model: EZ-9, MicroSense). From the measurement of the magnetic moment of the samples as a function of the Co thickness, it is possible to obtain the saturation magnetization $\left(M_{S}\right)$ and the effective thickness of Co layer $\left(t^{*}\right)$. The $M_{S}$ of Co in the $\mathrm{Pt} / \mathrm{Co} / \mathrm{MgO}(\mathrm{MgO} / \mathrm{Co} / \mathrm{Pt})$ sample is $1450 \pm 138(1430 \pm 151) \mathrm{emu} / \mathrm{cm}^{3}$; the effective thickness $\left(t^{*}\right)$ of $\mathrm{Pt} / \mathrm{Co} / \mathrm{MgO}(\mathrm{MgO} / \mathrm{Co} / \mathrm{Pt})$ is $t-0.64 \pm 0.01(t-0.14 \pm 0.05) \mathrm{nm}$, where $t$ is the deposited Co thickness. The effective anisotropy energy $\left(K_{\text {eff }}\right)$ is calculated by comparing perpendicular and in-plane $M-H$ hysteresis loops. Using the $K_{\text {eff }} \cdot t^{*}$ versus $t^{*}$ plot (Figure S2), we obtain the interface anisotropy energy $\left(K_{i}\right)$ from the $y$ intercept of a linear fit. The $K_{i}$ of $\mathrm{Pt} / \mathrm{Co} / \mathrm{MgO} \quad(\mathrm{MgO} / \mathrm{Co} / \mathrm{Pt})$ is $1.11 \pm 0.18(0.19 \pm 0.05) \mathrm{erg} / \mathrm{cm}^{2}$. It turns out that the 
$\mathrm{MgO} / \mathrm{Co} / \mathrm{MgO}$ sample has a negligible surface anisotropy without annealing, and the $\mathrm{Pt} / \mathrm{Co}$ and $\mathrm{Co} / \mathrm{Pt}$ are dominant interfaces for the interface anisotropy.

\section{Frequency shift from the interface anisotropy, $\Delta f_{\mathrm{ks}}$}

In the MSSW mode, the propagating spin wave is partially localized on either the top or bottom surface of ferromagnetic layer depending on the propagation direction. A substantial difference of surface anisotropy between the $\mathrm{Pt} / \mathrm{Co}$ and $\mathrm{Co} / \mathrm{MgO}$ interfaces could result in a considerable frequency shift even without DMI. We assume that the spin-wave amplitude in the Damon-Eshbach mode (with $+H$ ) decreases exponentially in the $z$ direction. The explicit expression for the spin wave amplitude $m^{ \pm}$is given as $m^{+}=e^{-|k| z}$ and $m^{-}=e^{-|k|(t-z)}$, where $k$ is the wave vector and $t$ is the thickness of ferromagnetic layer. The top interface of ferromagnetic layer corresponds to $z=0$. The effective anisotropy field is calculated from mean-field approach by weighing the relative amplitude of spin wave $\left(m^{ \pm}\right)$as follows [2];

$$
H_{A}^{e f f}=\frac{\int H_{A}^{i n t}(t) m^{ \pm}(t) d t}{\int m^{ \pm}(t) d t},
$$

where $H_{A}^{\mathrm{int}}=2 K_{i} / t_{a} M$ and $K_{\mathrm{i}}$ is the interface magnetic anisotropy, $t_{a}$ is the thickness of Co monolayer. Taking into account the anisotropy field, the frequency shift $\left(\Delta f_{k s}=f^{+}-f^{-}\right)$ induced by the surface anisotropy is then calculated from the spin wave dispersion relation;

$$
f^{ \pm}=\frac{\gamma}{2 \pi}\left[\sqrt{\left(H+4 \pi M_{s} P_{k}\right)\left(H-H_{A, t o p}^{e f f}-H_{A, \text { bottom }}^{e f f}+4 \pi M_{s}\left(1-P_{k}\right)\right)}\right]
$$

where $P_{k}=1-[1-\exp (-|k| t)] / k \mid t$. Figure S3 shows the calculated $\Delta f_{\mathrm{ks}}$ (hatched region) and measured $\Delta f$ (squares) of a $\mathrm{Pt} / \mathrm{Co}(20) / \mathrm{MgO}$ sample with $k=5.23 \mu \mathrm{m}^{-1}$. It turns out that the calculated $\Delta f_{\mathrm{ks}}$ is much smaller than the measured $\Delta f$. 


\section{The effect of interface anisotropy on the spin wave resonance frequency}

We have investigated the effect of interfacial anisotropy on the spin wave resonance frequency. Equation (2) implies that a large anisotropy field $\left(H_{A}\right)$, or a strong interfacial anisotropy, lowers the spin wave frequency $(f)$. In Fig. S4, we show the measured spin wave frequencies of the $\mathrm{Pt} / \mathrm{Co}(20) / \mathrm{MgO}, \mathrm{MgO} / \mathrm{Co}(20) / \mathrm{Pt}$, and $\mathrm{MgO} / \mathrm{Co}(20) / \mathrm{MgO}$ samples with a $k_{\mathrm{M}}$ of $7.85 \mu^{-1}$ as a function of external magnetic field. The $\mathrm{Pt} / \mathrm{Co}(20) / \mathrm{MgO}$ sample with a relatively strong interfacial anisotropy showed a lower spin wave frequency in comparison with those of $\mathrm{MgO} / \mathrm{Co}(20) / \mathrm{Pt}$ and $\mathrm{MgO} / \mathrm{Co}(20) / \mathrm{MgO}$ samples. Using Eq. (2) with $H=+500$

Oe, $\gamma=1.77 \times 10^{-2} \mathrm{Oe}^{-1} \cdot \mathrm{ns}^{-1}, M_{\mathrm{s}}=1430 \mathrm{emu} / \mathrm{cm}^{3}, H_{A}\left(=2 K_{\mathrm{i}} / t M_{\mathrm{s}}\right), t=20 \mathrm{~nm}$, and $k=7.85 \mu \mathrm{m}^{-}$ 1 , it is possible to calculate the corresponding spin wave frequency of the $\mathrm{Pt} / \mathrm{Co} / \mathrm{MgO}$, $\mathrm{MgO} / \mathrm{Co} / \mathrm{Pt}$, and $\mathrm{MgO} / \mathrm{Co} / \mathrm{MgO}$ samples. We find that the measured spin wave frequencies are well fitted to Eq. (2).

\section{Possible frequency modulation using the electrical field control of interface anisotropy}

The spin wave modulators depicted in Fig. 5a can be also operated by the electric field control of interfacial anisotropy. Considering the influence of $K_{i}$ in Eq. (2), we can calculate the frequency of propagating spin wave as a function of voltage bias $\left(V_{\text {app }}\right)$. Figure S5 shows the calculated results for the frequency modulation as a function of $\mathrm{d} K_{\mathrm{i}} / \mathrm{d} V_{\text {app }}$. It turns out that, when the $K_{i}$ is sufficiently controlled by $V_{\text {app }}$, a sizable frequency modulation is expected, and the frequency modulation has been enhanced with reducing the thickness of Co layer. With a 2-nm-thick Co layer, the spin-wave frequency is modulated about $24 \%$ with varying the $K_{i}$ from 1.1 to $0 \mathrm{erg} / \mathrm{cm}^{2}$. 


\section{Finite size effect on the DMI measurements}

We find that the finite-size effect, especially at the edges, on the spin wave propagation does not affect our estimation of DMI as explained below. To check this edge effect, we conducted micromagnetic simulation with modeling parameters as follows: the stripe length $=1.2 \mu \mathrm{m}$, the stripe width $=1.0,1.5,2.0$, and $8.0 \mu \mathrm{m}$ with the DMI boundary condition [3], the Co thickness $=20 \mathrm{~nm}$ with a unit cell size of $10 \times 10 \times 20 \mathrm{~nm}^{3}$, the damping constant $\alpha=0.01$, the saturation magnetization $M_{S}=1400 \mathrm{emu} / \mathrm{cm}^{3}$, the exchange stiffness constant $A_{e x}=3.0 \times 10^{-6}$ $\mathrm{erg} / \mathrm{cm}$, the DM constant $D=0.2 \mathrm{erg} / \mathrm{cm}^{2}$, the external field along width direction $H_{\text {ext }}=1000$ Oe, and the antenna frequency $f_{\text {ant }}=26 \mathrm{GHz}$. Because the actual sample dimension is too large to calculate with limited computing time, we use the lateral cell size $(=10 \mathrm{~nm})$ larger than the exchange length $(\sim 5 \mathrm{~nm})$. We believe this choice of the cell size does not alter the main conclusion in this response because the magnetization gradient in spin wave dynamics is extremely small. By the same reason, we choose a somewhat higher frequency than that used in our experiment. All other parameters are chosen to mimic the properties of bulk Co.

In Fig. S6, we show the equilibrium magnetization configurations of microstrip with various widths ((a) $1.0 \mu \mathrm{m}$, (b) $1.5 \mu \mathrm{m}$, and (c) $2.0 \mu \mathrm{m}$ ). In the figure, the arrows correspond to the in-plane ( $x y$-plane) magnetization vectors and the color code corresponds to the $y$ component of magnetization $\left(M_{y}\right)$. We observe the magnetization tilting at the edges as a consequence of demagnetization fields. We also observe that the magnetization tilting in the thickness direction at the edges, which is induced by the DM boundary condition, is smaller than the tilting in $y$ direction because of the small DMI constant. The width of the edge-tilting region is about $100 \mathrm{~nm}$, and is found to be independent of the full width of microstrip.

In Fig. S7(a), we show a snapshot of spin wave propagation with an 8 - $\mu$ m-wide microstrip; this width is the same as the width of the sample used in the experiment. Fig. S7(b) shows the $M_{z}$-component averaged along the width direction. From this plot and the procedure 
explained in the main text, we are able to deduce the DM constant as $0.21 \mathrm{erg} / \mathrm{cm}^{2}$, which is close to the assumed DM constant in this simulation with high accuracy. This confirms that the edge effect is not so significant to change the measured DM constant in our experiments. Therefore, we conclude that, even with the edge-tilting of magnetization, our experimental methodology is able to estimate the DMI value correctly.

\section{Supporting References}

1. Vlaminck, V.; Bailleul, M. Physical Review B 2010, 81, (1), 014425.

2. Nembach, H. T.; Shaw, J. M.; Weiler, M.; Jué, E.; Silva, T. J. Nat Phys 2015, 11, 825.

3. Rohart, S.; Thiaville, A. Physical Review B 2013, 88, (18), 184422. 


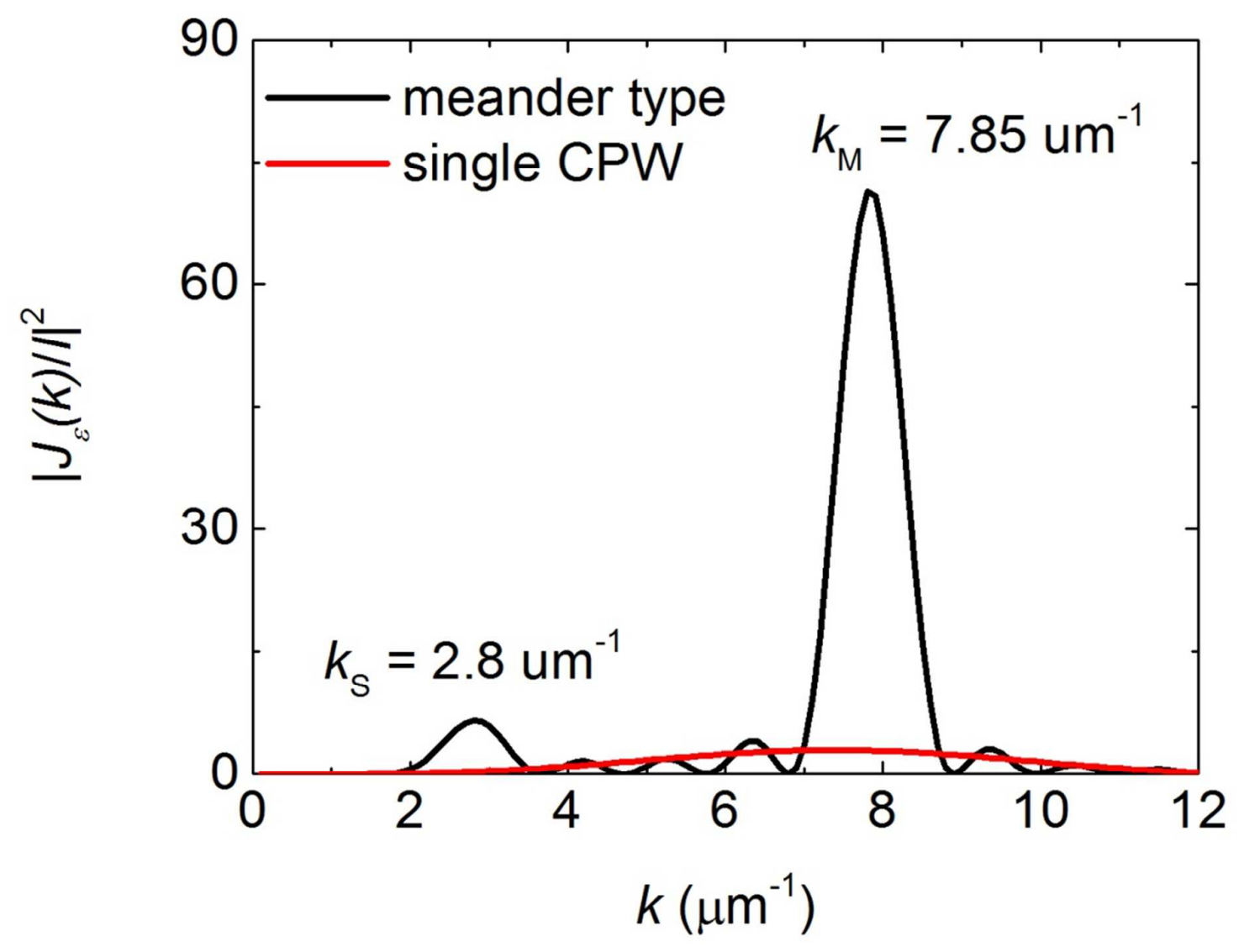

Figure S1: Fourier transform of current density $\left(\widetilde{j}_{\varepsilon}(k)\right)$ from a coplanar wave guide (CPW) consisting of five-segment meander lines (See Fig. 1b). The signal from a single CPW antenna (red line) is shown for comparison. 


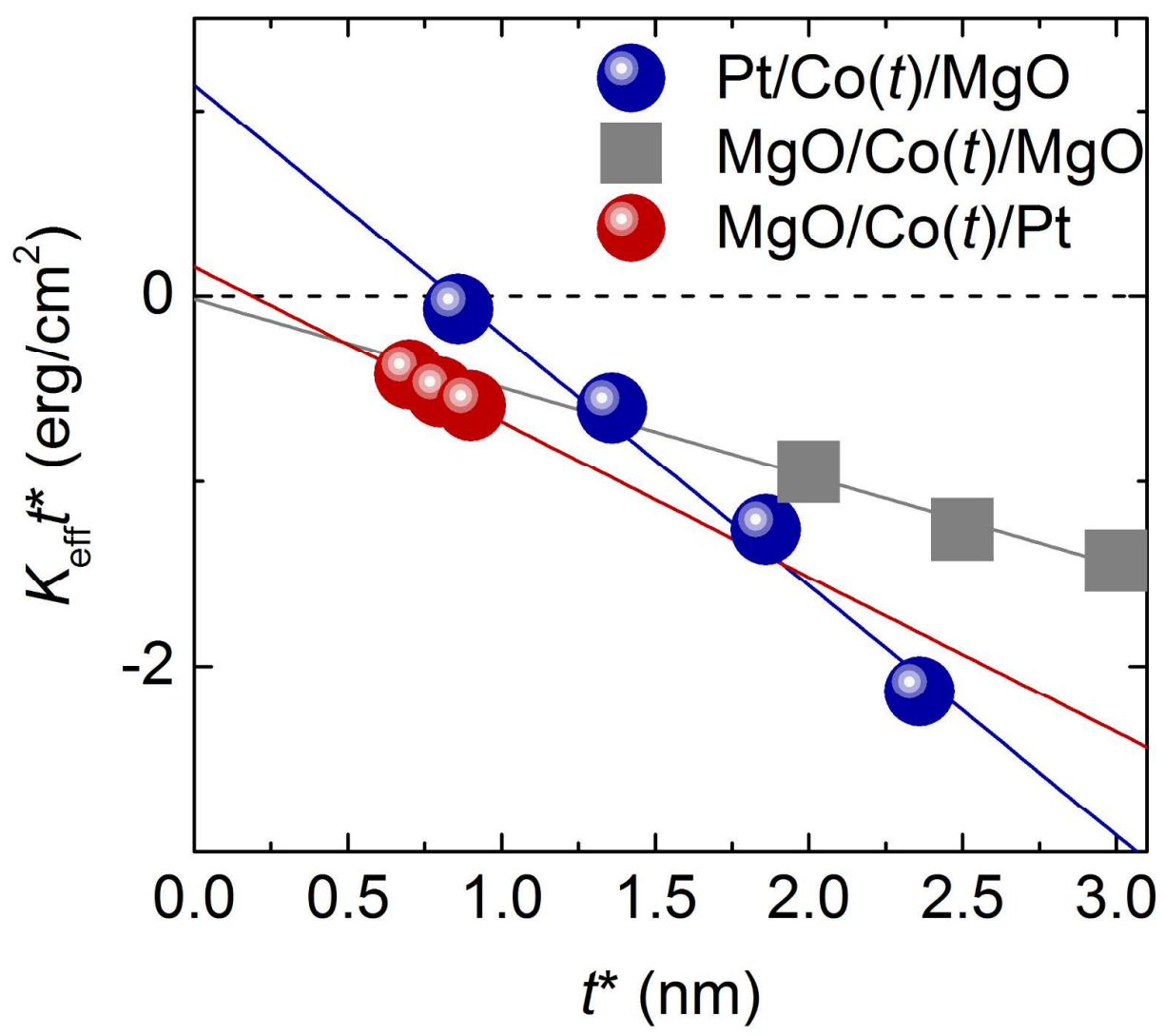

Figure S2: Effective anisotropy $\left(K_{\text {eff }}\right)$ multiplied by the effective Co thickness $\left(t^{*}\right)$ as a function of $t^{*}$. The interface anisotropy $\left(K_{i}\right)$ of the $\mathrm{Pt} / \mathrm{Co} / \mathrm{MgO}(\mathrm{MgO} / \mathrm{Co} / \mathrm{Pt}), 1.11(0.19)$ $\mathrm{erg} / \mathrm{cm}^{2}$, is determined from the $y$ intercept of the linear fit. 


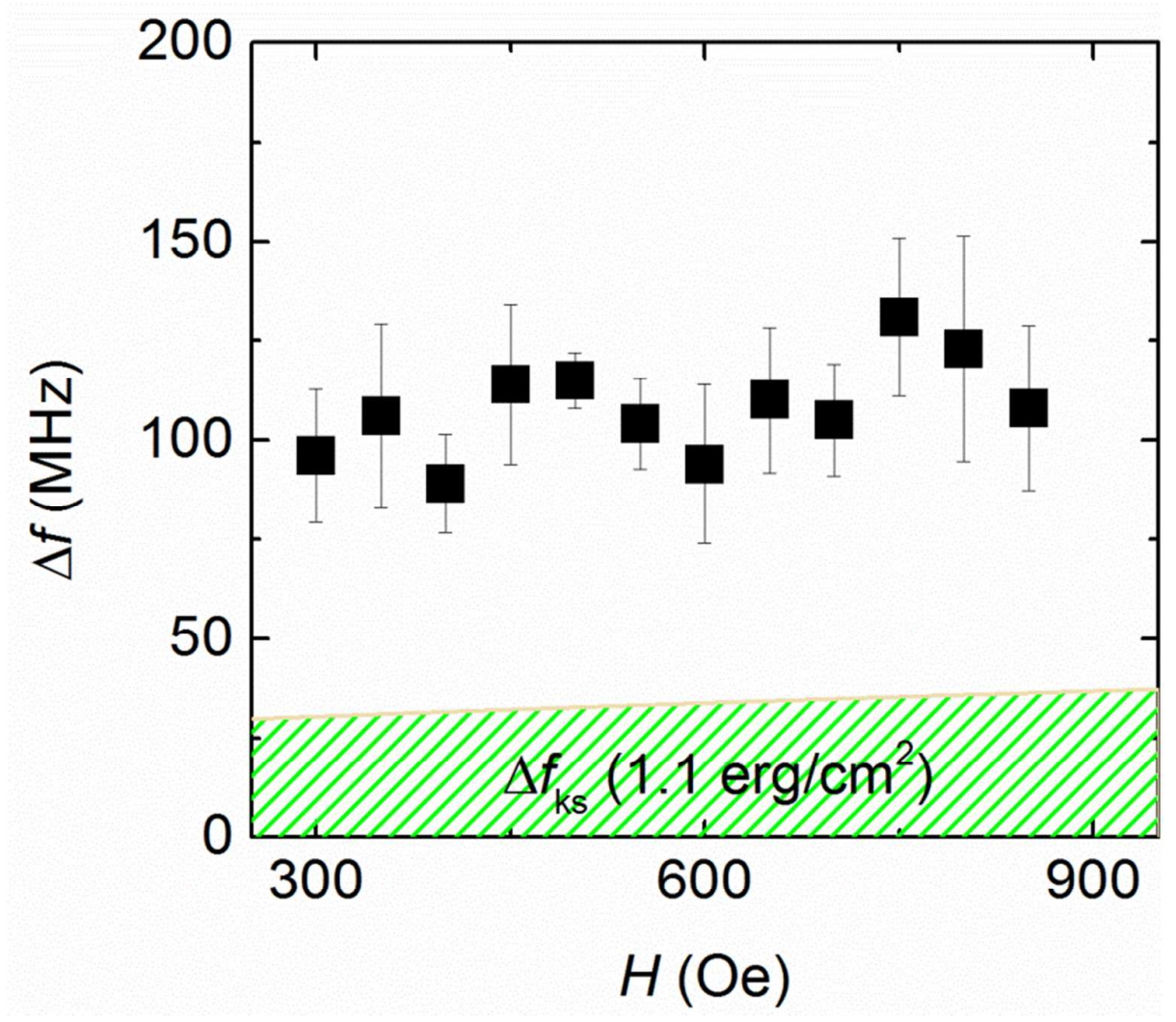

Figure S3: Measured $\Delta f$ (squares) and calculated $\Delta f_{\mathrm{ks}}$ (hatched region) of the $\mathrm{Pt} / \mathrm{Co}(20) / \mathrm{MgO}$ sample with $k=5.23 \mu \mathrm{m}^{-1}$. 


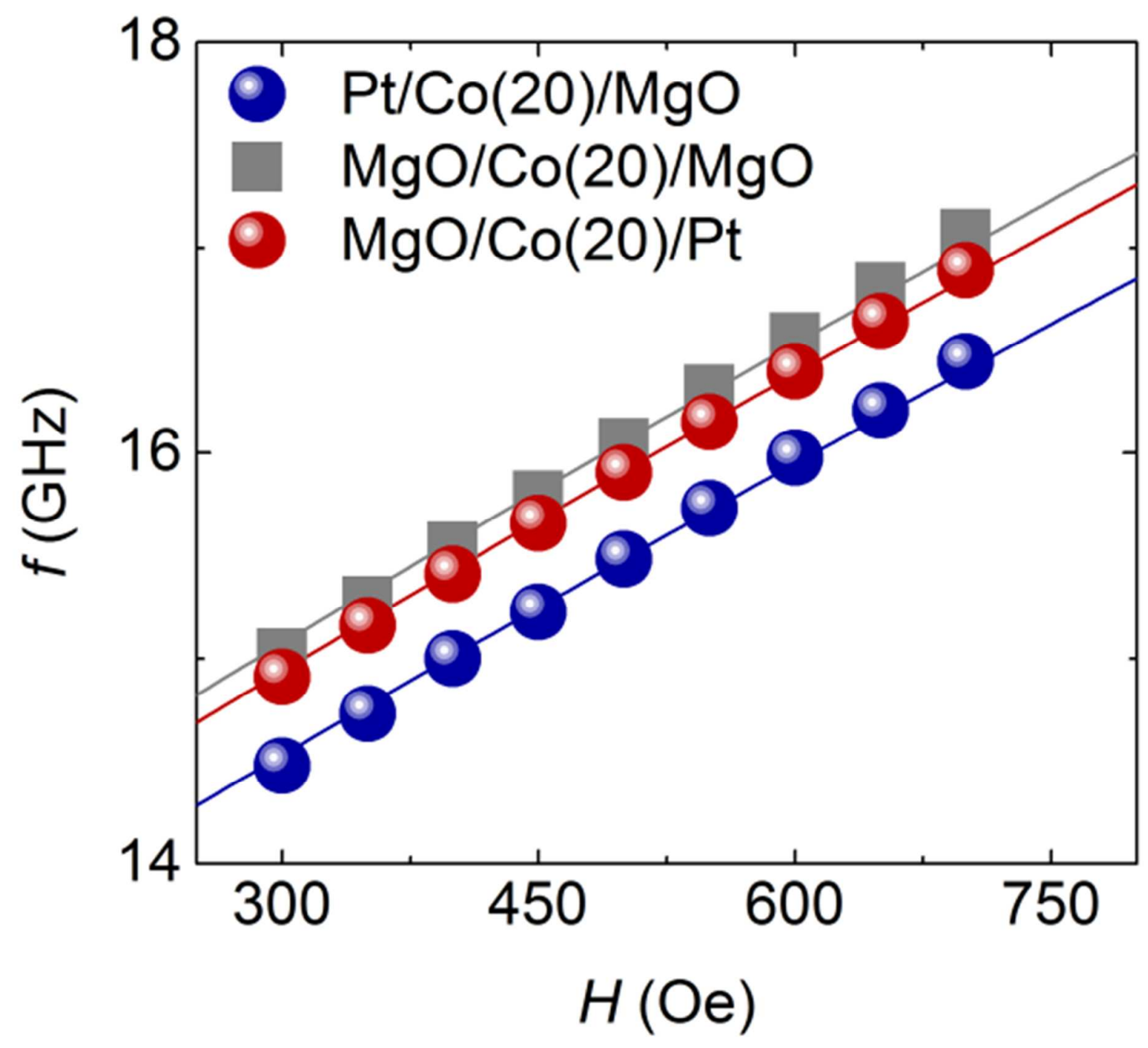

Figure S4: The measured spin wave resonance frequency (symbols) of the $\mathrm{MgO} / \mathrm{Co}(20) / \mathrm{Pt}$ and $\mathrm{MgO} / \mathrm{Co}(20) / \mathrm{MgO}$ samples as a function of external magnetic field. The solid line indicate a fit using Eq. (2) with $H=+500 \mathrm{Oe}, \gamma=1.77 \times 10^{-2} \mathrm{Oe}^{-1} \cdot \mathrm{ns}^{-1}, M_{\mathrm{s}}=1430 \mathrm{emu} / \mathrm{cm}^{3}, t$ $=20 \mathrm{~nm}$, and $k=7.85 \mu \mathrm{m}^{-1}$. 


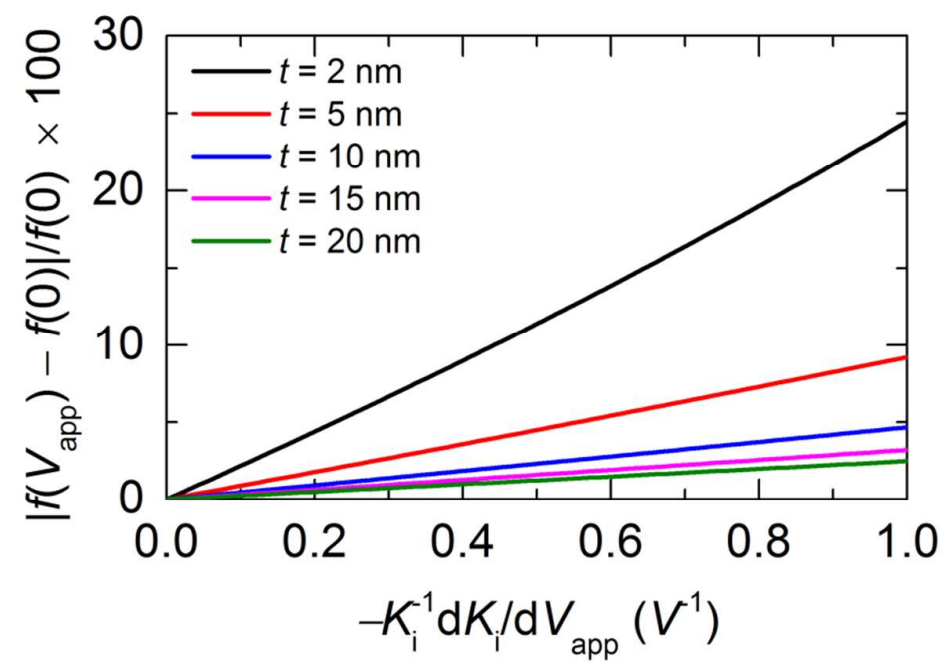

Figure S5: Frequency modulation as a function of $\mathrm{d} K_{\mathrm{i}} / \mathrm{d} V_{\text {app }}$. The spin-wave frequency $\left(f\left(V_{\text {app }}\right)\right)$ at the detector is calculated as a function of voltage bias ( $\left.V_{\text {app }}\right)$ using Eq. (S3) with following parameters: $H=+200 \mathrm{Oe}, M_{\mathrm{s}}=1430 \mathrm{emu} / \mathrm{cc}$, and $K_{\mathrm{i}}(0 \mathrm{~V})=1.1 \mathrm{erg} / \mathrm{cm}^{2}$. 


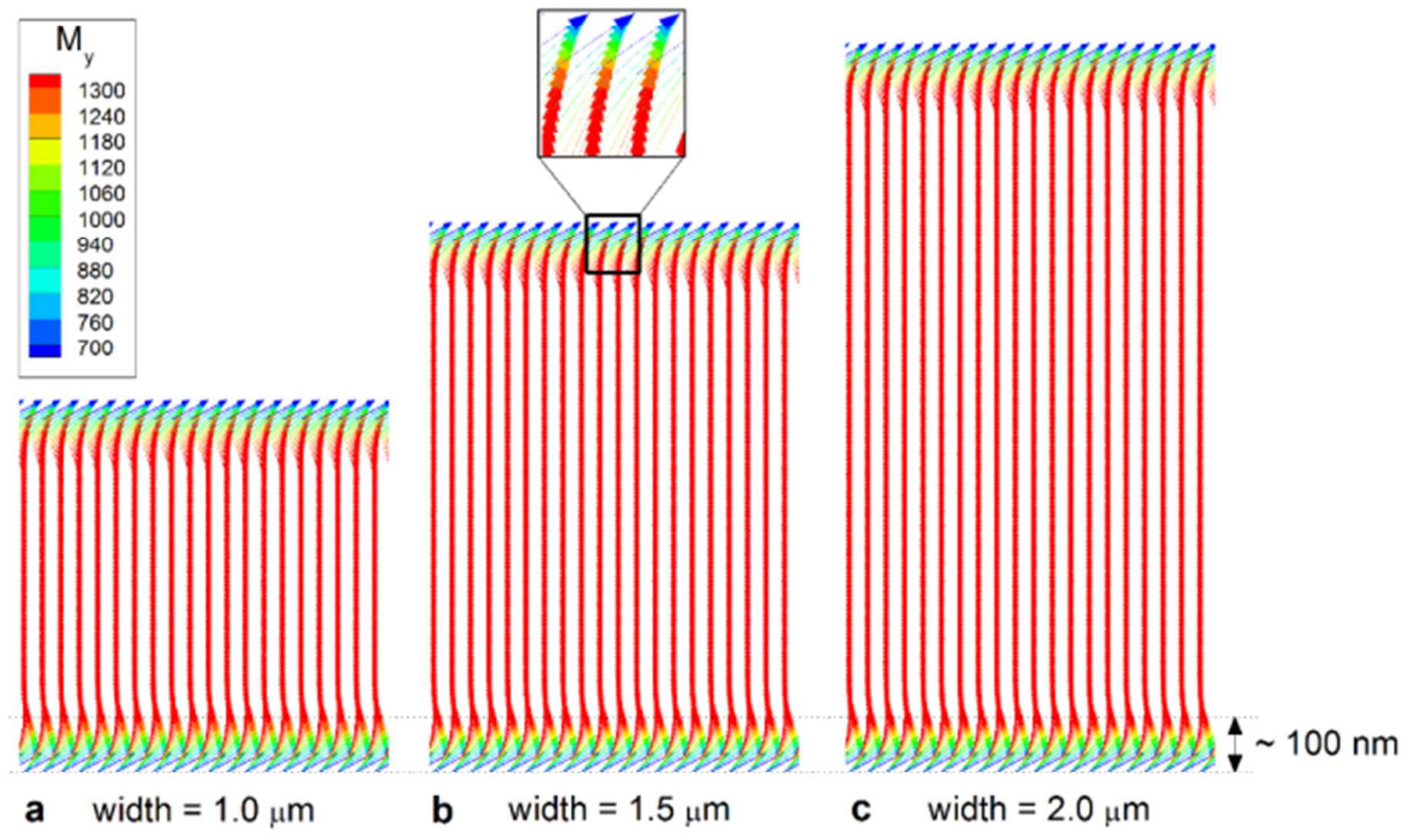

Figure S6: Magnetization configurations of microstrip with various widths (a) 1.0, (b) 1.5, (c) $2.0 \mu \mathrm{m}$. The arrows correspond to the magnetization vector in the plane, and the color code corresponds to the $y$-component of magnetization $\left(M_{y}\right)$. The unit of $M_{y}$ is emu $/ \mathrm{cm}^{3}$. 


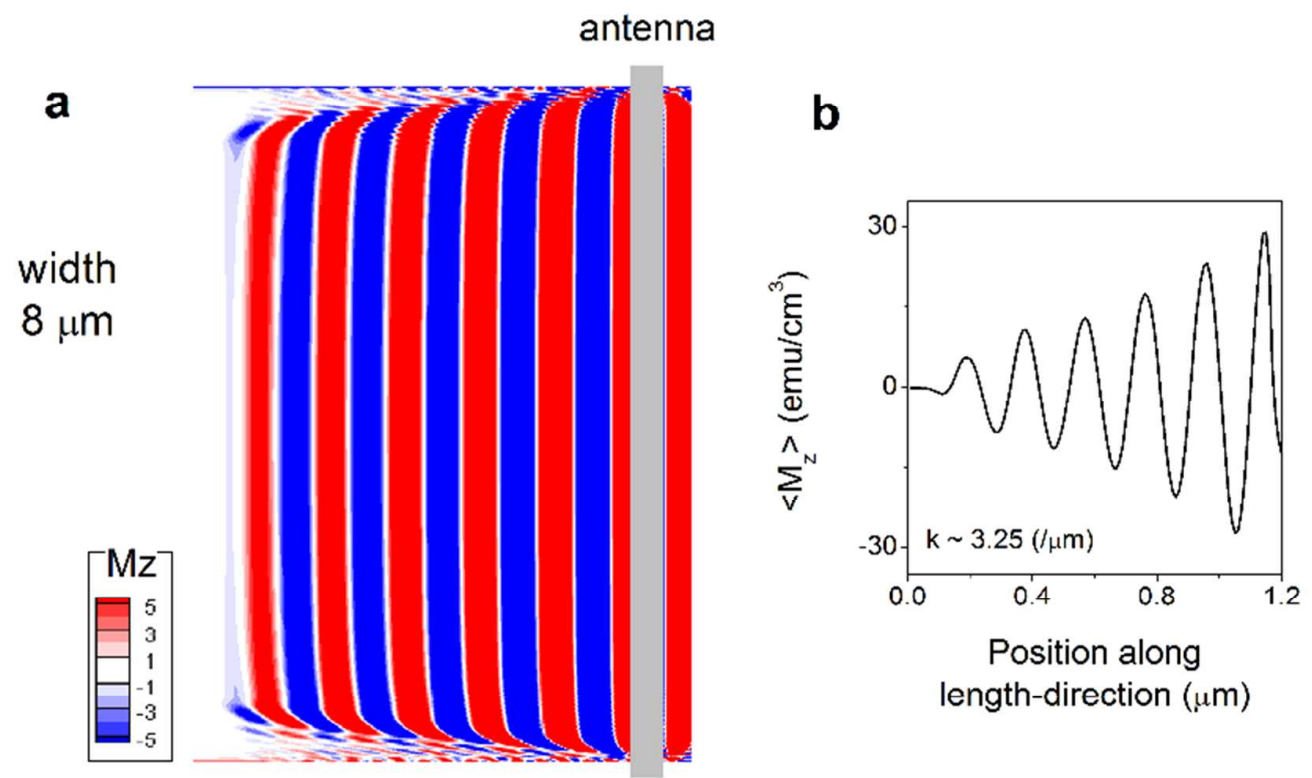

Figure S7: (a) A snapshot of spin wave propagation in an 8- $\mu \mathrm{m}$-wide microstrip. The color code indicates the $z$-component of magnetization $\left(M_{z}\right)$. (b) $M_{z}$ component averaged along the width direction. 